\title{
STATIC CURRENT-SHEET MODELS OF QUIESCENT PROMINENCES
}

\author{
F. $\mathrm{Wu}^{1}$ \\ B. C. Low \\ High Altitude Observatory \\ National Center for Atmospheric Research ${ }^{2}$
}

\section{INTRODUCTION}

A particular class of theoretical models idealize the prominence to be a discrete flat electriccurrent sheet suspended vertically in a potential magnetic field. The weight of the prominence is supported by the Lorentz force in the current sheet. These models can be extended to have curved electric-current sheets and to vary three-dimensionally.

The equation for force balance is

$$
\frac{1}{4 \pi}(\nabla \times \mathbf{B}) \times \mathbf{B}-\nabla p-\rho g \hat{\mathbf{z}}=0
$$

Using Cartesian coordinates we take, for simplicity, a uniform gravity with constant acceleration $g$ in the the direction $-\hat{\mathbf{z}}$. If we are interested not in the detailed internal structure of the prominence, but in the global magnetic configuration around the prominence, we may take the prominence plasma to be cold. Let us consider how such equilibrium states can be constructed. To simplify the mathematical problem, suppose there is no electric current in the atmosphere except for the discrete currents in the cold prominence sheet. Let us take the plane $z=0$ to be the base of the atmosphere and restrict our attention to the domain $z>0$. The task we have is to solve for a magnetic field which is everywhere potential except on some free surface $S$, subject to suitable boundary conditions. The surface $S$ is determined by requiring that it possesses a discrete electric current density such that the Lorentz force on it is everywhere vertically upward to balance the weight of the material $m(S)$. Since the magnetic field is potential in the external atmosphere, the latter is decoupled from the magnetic field and its plane parallel hydrostatic pressure and density can be prescribed quite separately. Instead of solving this difficult free boundary problem, classes of illustrative models can be constructed by solving for particular solutions to Ampere's equation for a discrete current density $\mathrm{J}(S)$ in a potential magnetic field such that the Lorentz force at the surface $S$ is everywhere vertically upward. Then we can define the equilibrium mass distribution $m(S)$ having just the right weight to balance the Lorentz force.

\section{TWO DIMENSIONAL MODELS}

If the magnetic field has no $x$-component and is everywhere potential except on the sheet, the magnetic field can be represented in terms of an analytic function of the complex variable $w=$ $y+i z$. Priest and Raadu (1975) have given an example for a plane vertical current sheet not threaded by magnetic lines of force. The electric current in the sheet is directed in the $x$ direction. We can superpose an additional dipole source in the region $z<0$ to this current sheet model. The additional magnetic field introduced at the current sheet is horizontal and the Lorentz force that results is everywhere upward (see Figure 1). Evaluating the Lorentz force on the current sheet, we get the mass distribution on the current sheet. By changing the strength of the additional dipole source we can get both the so-called Kuperus-Raadu model and the Kippenhahn-Schluter model (Kippenhahn and Schluter 1957, Kuperus and Raadu 1974).

\footnotetext{
${ }^{1}$ Permanent address: Beijing Astronomical Observatory, Academia Sinica, China.

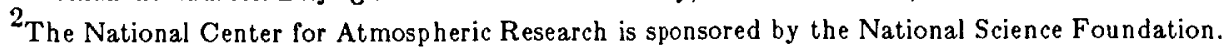




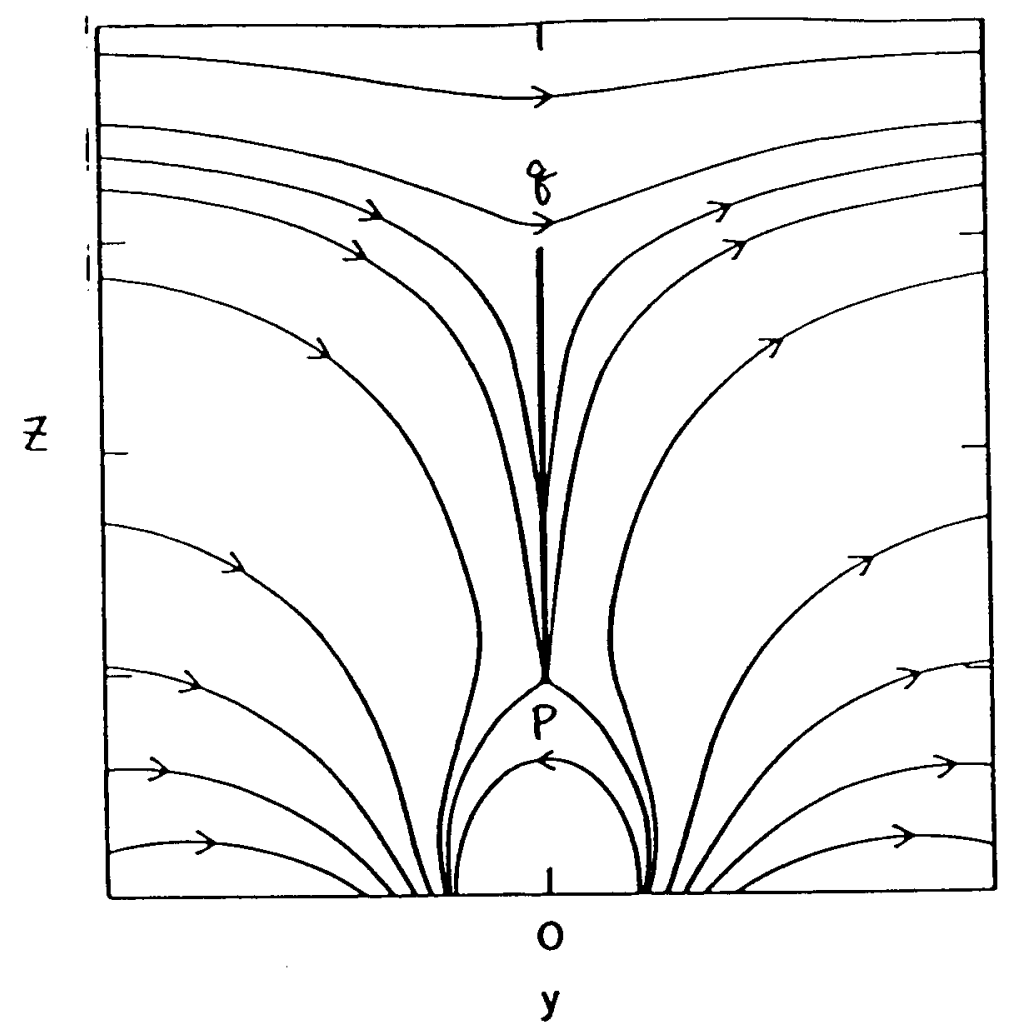

Figure 1. Field lines in the $y-z$ plane obtained by superposing an additional dipole source located in the $z<0$ plane with the finite current sheet which runs along the $y$ axis, extending from $z=p$ to $z=q$.

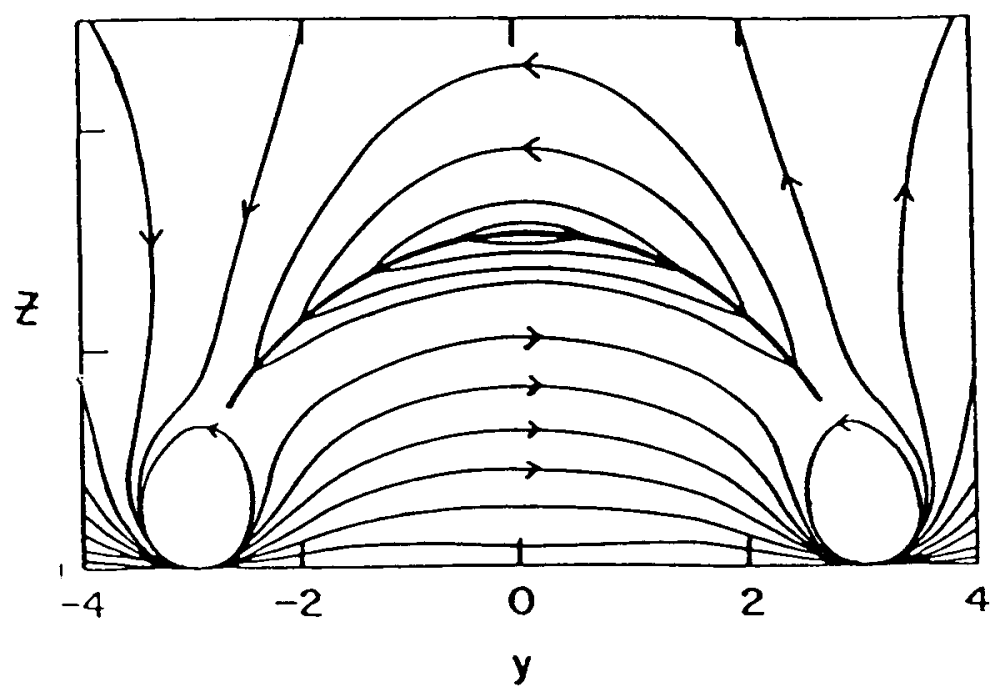

Figure 2. Field lines in the $y-z$ plane obtained by superposing a circular current sheet denoted by the thick arc with an additional potential field which is strictly horizontal everywhere on the current sheet. 
A similar construction can be carried out if explicit solutions for the curved current sheets are given. Solutions for current sheets which are circular in shape have been obtained by $\mathrm{Hu}$ and Low (1982) and Hu (1984). To these solutions, the superposed potential field should be strictly horizontal on the circular sheet in order that the Lorentz force on the circular sheet is everywhere vertically upwards (see Figure 2). Then a certain amount of mass can be supported by the Lorentz force. Several examples can be found in the paper by Wu and Low (1986). It is interesting to note that the tension force at the current sheet is no longer vertical, and there is a discontinuity in the magnetic pressure at the current sheet. It is the combination of the pressure force, normal to the current sheet, and the oblique tension force that supports the weight. This is a novel feature not found in the case of a vertical current sheet.

\section{THREE-DIMENSIONAL MODELS}

As a step of further sophistication, the superposed potential field may be allowed to vary in three dimensions. We present two simple examples obtained by superposing the vertical plane current sheet solution given by Priest and Raadu (1975), with a periodic three dimensional potential field in one case, and with a non-periodic three dimensional potential field due to a finite number of dipole sources in the other (see $\mathrm{Wu}$ and Low 1986). The $x$ variation in these models provides a natural length scale over which a total energy can be meaningfully defined. When a quiescent prominence is observed in $H_{\alpha}$ at the solar limb, it sometimes appears like a hedgerow with a repetitious structure. We may take the periodic solution to model such a structure. For the non-periodic solution, the distance in the $x$ direction over which the dipole sources are distributed is a characteristic length of the prominence. Let us insert parametric values characteristic of prominences into these two sets of solutions to examine the energy content.

If we neglect the energy due to the plane parallel hydrostatic atmosphere outside the electric current sheet, the total energy consists of the magnetic energy and the gravitational potential energy of the suspended mass. Put the lower edge of the current sheet at a height of about $2 \times 10^{4} \mathrm{~km}$ and the top edge of the current sheet at about $5 \times 10^{4} \mathrm{~km}$ (see, e.g., TandbergHanssen 1974). By choosing suitable constants in the periodic solution, the normal field at the base of corona is about $80 \mathrm{G}$. If we take each period in the $x$ direction to be a single hedgerow component of a prominence, the length in that direction is about $6 \times 10^{4} \mathrm{~km}$. With these parameters set and using a uniform gravitational acceleration of $g=1.6 \times 10^{4} \mathrm{~cm} \mathrm{~s}^{-2}$, the total mass in one period is of the order of $2 \times 10^{14} \mathrm{gm}$. This total mass is equal to the total mass of a prominence which has the same length, a thickness of $5 \times 10^{3} \mathrm{~km}$ and an average density of $10^{10}$ protons $\mathrm{cm}^{-3}$, in agreement with observations (Tandberg-Hanssen 1974). The free magnetic energy is about $3 \times 10^{28} \mathrm{erg}$, which is the same order of magnitude as the gravitational potential energy of $1.3 \times 10^{28} \mathrm{erg}$. Using suitable parameters for the non-periodic case the normal field at the base of the corona is about $54 \mathrm{G}$. Let the prominence have a length of $2 \times 10^{5} \mathrm{~km}$, the total mass in the current sheet is about $6.4 \times 10^{14} \mathrm{gm}$. The gravitational potential energy is about $4 \times 10^{28} \mathrm{erg}$. The free magnetic energy is about $9 \times 10^{27} \mathrm{erg}$. In these above examples, the gravitational potential energy turns out to be larger than the magnetic free energy. It follows that if the prominence erupts, draining a fraction of the suspended mass to the base of the corona, the gravitational potential energy associated with the drained material may have an important role in driving the remaining mass outward from the Sun.

It will be interesting to pursue the following further development of the current sheet model. To support the weight of the current sheet, the electric current must be horizontal in order that the Lorentz force associated with it is vertical to balance the force of gravity. This implies that the electric current must either flow horizontally to infinity, such as in the models shown in Figure 1 and 2, or else close upon itself in horizontal loops. It will be interesting to generate classes of solutions corresponding to the latter possibility. A simple case would be a solution with current sheets in the form of surfaces of revolution about a vertical axis of symmetry. More general are the solutions with current sheets that are of complex geometries and closing upon themselves in a localized region of the atmosphere. In the realistic prominence, electric currents may actually flow along magnetic field lines to close themselves in the photosphere; see the threedimensional solutions of Low (1984). Finally, we point out that the availability of explicit 
equilibrium solutions opens up the opportunity of studying the linear hydromagnetic stability of magnetically suspended mass sheets. The stability of the solutions in Figure 1 and 2 will be taken up elsewhere (Wu 1986).

\section{REFERENCES}

Hu,Y. Q., 1984, Acta Astrophysica Sinica, 4, 14.

Hu, Y. Q., and Low, B.C., 1982, Solar Phys., 81, 107.

Kippenhahn, R., and Schluter, A., 1957, Z.Astrophys., 43, 36.

Kuperus, M., and Raadu, M. A., 1974, Astron. Astrophys., 31189.

Low, B. C., 1984, Ap. J., 277, 415.

Priest, E. R., and Raadu, M. A., 1975, Solar Phys., 43, 177.

Tandberg-Hanssen, E., 1974, Solar Prominences, (Dordrecht: Reidel)

Wu, F., 1986, (in preparation)

Wu, F., and Low, B. C., 1986, AP. J., (submitted) 